\title{
DYNAMICAL AND TECHNOLOGICAL CONSEQUENCES OF MULTIPLE ISOLAS OF STEADY STATES IN A CATALYTIC FLUIDISED-BED REACTOR
}

\author{
Katarzyna Bizon* \\ Cracow University of Technology, Department of Chemical and Process Engineering, 30-155 \\ Kraków, ul. Warszawska 24, Poland
}

\begin{abstract}
Steady-state characteristics of a catalytic fluidised bed reactor and its dynamical consequences are analyzed. The occurrence of an untypical steady-state structure manifesting in a form of multiple isolas is described. A two-phase bubbling bed model is used for a quantitative description of the bed of catalyst. The influence of heat exchange intensity and a fluidisation ratio onto the generation of isolated solution branches is presented for two kinetic schemes. Dynamical consequences of the coexistence of such untypical branches of steady states are presented. The impact of linear growth of the fluidisation ratio and step change of the cooling medium temperature onto the desired product yield is analyzed. The results presented in this study confirm that the identification of a region of the occurrence of multiple isolas is important due to their strong impact both on the process start-up and its control.
\end{abstract}

Keywords: catalytic fluidised-bed reactor, steady states, non-linear analysis, multiplicity, multiple isolas

\section{INTRODUCTION}

A phenomenon of generation of single isolas, i.e. isolated closed branches of steady states has been demonstrated a number of times in chemical engineering systems, both in theoretical and experimental works. Their occurrence has been observed in very simple systems such as non-adiabatic continuously stirred tank reactors (Berezowski, 2002; Uppal et al., 1976), tubular heterogeneous reactors (Berezowski, 2000) as well as in more complex catalytic processes carried out in fluidised-bed reactors (Ajbar et al., 2001). Several works, e.g. (Pinto and Ray, 1995), report on the possibility of the occurrence of so-called multiple isolas of steady states. In such a case several closed branches of steady states co-existing with open steady-state branches are observed in a bifurcation diagram. In the work cited above the authors also described scenarios of generation and disappearance of the isolas. Pinto and Ray (1995) demonstrated the possibility of occurrence of two isolas of steady states in the bifurcation diagram of the process of copolymerization of vinyl acetate and methyl methacrylate in tertbutanol. The isolas are located next to each other, that is in different ranges of the selected bifurcation parameter, in this case a residence time. In this study such isolas will be referred to as the first type isolas. Despite an extensive body of literature concerning the steady-state analysis of chemically reactive systems, the possibility of co-existence of two isolas in the same range of the bifurcation parameter, i.e. one isola above the other, and an open branch of steady states was demonstrated only in a couple of works. The possibility of such a phenomenon was mentioned in (Zahn, 2012) and in the earlier study of the author of this work (Bizon, 2016). These isolas will be referred to as the second type isolas. 
This study demonstrates that such an untypical structure of steady-states can develop in catalytic fluidised-bed reactors. A single chemical reaction and a system of two consecutive reactions were used to exemplify the occurrence of multiple isolas. The impact of a heat exchange intensity and a fluidisation ratio onto the generation of isolated solution branches was evaluated. Dynamical and technological consequences of the existence of multiple isolas of steady states were analysed.

\section{MATHEMATICAL MODEL OF A CATALYTIC FLUIDISED-BED REACTOR}

Let us consider the chemical processes taking place in a catalytic fluidised-bed reactor, namely:

- a single irreversible first order chemical reaction proceeding according to a scheme $\mathrm{A} \stackrel{k_{1}}{\longrightarrow} \mathrm{P}$;

- a system of consecutive irreversible first order chemical reactions: $\mathrm{A} \stackrel{k_{1}}{\longrightarrow} \mathrm{P} \stackrel{k_{2}}{\longrightarrow} \mathrm{R}$, where $\mathrm{P}$ is a desired product, whereas $\mathrm{R}$ is an undesired product.

For the process (a) the reaction rate with respect to the component A is $r_{\mathrm{A}}=r_{1}$, whereas for the system of reactions in series the rates with respect to $\mathrm{A}$ and $\mathrm{P}$ are, respectively, $r_{\mathrm{A}}=r_{1}$ and $r_{\mathrm{P}}=r_{1}-r_{2}$, where

$$
r_{i}=C_{j} \cdot k_{0 i} \exp \left(-\frac{E_{i}}{R T}\right), i=1,2 ; j=\mathrm{A}, \mathrm{P}
$$

A two-phase bubbling bed model, modified by Tabiś (Tabiś, 2001) was employed to describe hydrodynamics of a fluidised bed. The main assumption of the model is that the bed consists of an emulsion phase and a bubble phase with the mass and heat transfer coefficients between the phases determined as resultant quantities according to the three-phase model of Kunii and Levenspiel (1991). The other assumptions are:

- perfect mixing and negligible mass and heat transfer resistance between the gas and the catalyst particles in the emulsion phase;

- plug flow of the gas and quasi-steady state character of the bubble phase.

According to these assumptions the dynamic mass and heat balance equations in the emulsion phase can be written as:

$$
\begin{gathered}
\frac{d \eta_{\mathrm{A}}^{e}}{d t}=a_{2}\left(\eta_{\mathrm{A} f}-\eta_{\mathrm{A}}^{e}\right)+\varphi_{1}\left(\eta_{\mathrm{A}}^{e}\right)-a_{3} \cdot \widetilde{r}_{\mathrm{A}}\left(\eta_{\mathrm{A}}^{e}, T^{e}\right)=f_{1}\left(\eta_{\mathrm{A}}^{e}, T^{e}\right) \\
\frac{d \eta_{\mathrm{P}}^{e}}{d t}=a_{2}\left(\eta_{\mathrm{P} f}-\eta_{\mathrm{P}}^{e}\right)+\varphi_{2}\left(\eta_{\mathrm{P}}^{e}\right)+a_{3} \cdot \widetilde{r}_{\mathrm{P}}\left(\eta_{\mathrm{A}}^{e}, \eta_{\mathrm{P}}^{e}, T^{e}\right)=f_{2}\left(\eta_{\mathrm{A}}^{e}, \eta_{\mathrm{P}}^{e}, T^{e}\right) \\
\left.\frac{d T^{e}}{d t}=a_{5}\left(T_{f}-T^{e}\right)-\varphi_{3}\left(T^{e}\right)+a_{4} \sum_{i=1}^{2}\left(-\Delta h_{i}\right) \cdot \widetilde{r}_{i}\left(\eta_{\mathrm{A}}^{e}, \eta_{\mathrm{P}}^{e}, T^{e}\right)-Q_{1}\left(T^{e}-T_{q}\right)=f_{3}\left(\eta_{\mathrm{A}}^{e}, \eta_{\mathrm{P}}^{e}, T^{e}\right)\right)
\end{gathered}
$$

where a dimensionless concentration of a $j^{\text {th }}$ reactant $\eta_{j}$ is defined as

$$
\eta_{j}=\frac{C_{j}}{C_{\mathrm{A} r e f}}, j=\mathrm{A}, \mathrm{P} \text { where } C_{\mathrm{A} r e f}=\frac{P \cdot y_{\mathrm{A} f}}{R \cdot T_{f}}
$$

whereas expressions defining the reaction rates in a function of dimensionless concentration $\eta_{j}^{e}$ and temperature $T^{e}$ in the emulsion are given by

$$
\begin{gathered}
\widetilde{r}_{i}=k_{i} \eta_{j}^{e}=k_{0 i} \exp \left(-\frac{E_{i}}{R T^{e}}\right) \cdot \eta_{j}^{e}, i=1,2 ; j=\mathrm{A}, \mathrm{P} \\
\widetilde{r}_{\mathrm{A}}=\widetilde{r}_{1} ; \quad \widetilde{r}_{\mathrm{A}}=\widetilde{r}_{1}-\widetilde{r}_{2}
\end{gathered}
$$


The functions $\varphi_{1}\left(\eta_{\mathrm{A}}^{e}\right), \varphi_{2}\left(\eta_{\mathrm{P}}^{e}\right)$ and $\varphi_{3}\left(T^{e}\right)$ in Equations (2) - (4) are defined as

$$
\begin{gathered}
\varphi_{1}\left(\eta_{\mathrm{A}}^{e}\right)=\frac{B_{1 \mathrm{~A}}}{B_{3 \mathrm{~A}}}\left(\eta_{\mathrm{A}}^{e}-\eta_{\mathrm{Af}}\right) \cdot\left[\exp \left(-B_{3 \mathrm{~A}}\right)-1\right] \\
\varphi_{2}\left(\eta_{\mathrm{P}}^{e}\right)=\frac{B_{1 \mathrm{P}}}{B_{3 \mathrm{P}}}\left(\eta_{\mathrm{P}}^{e}-\eta_{\mathrm{P} f}\right) \cdot\left[\exp \left(-B_{3 \mathrm{P}}\right)-1\right] \\
\varphi_{3}\left(T^{e}\right)=\frac{B_{2}}{B_{4}+Q_{2}}\left[Q_{2}\left(T^{e}-T_{q}\right)+\left(T_{f}-\frac{B_{4} T^{e}+Q_{2} T_{q}}{B_{4}+Q_{2}}\right)\left(\exp \left(-\left(B_{4}+Q_{2}\right)\right)-1\right)\right]
\end{gathered}
$$

The parameters in Equations (2) - (4) and in expressions (7) - (9) are calculated as follows

$$
\begin{gathered}
a_{1}=\frac{\rho_{z} c_{z}}{\rho_{g} c_{g}} ; \quad a_{2}=\frac{u_{e}}{H} ; \quad a_{3}=\frac{1-\varepsilon_{m f}}{\varepsilon_{m f}} ; \quad a_{4}=\frac{\left(1-\varepsilon_{m f}\right) C_{\mathrm{Aref}}}{\left(1-\varepsilon_{m f}+\varepsilon_{m f} / a_{1}\right) \rho_{z} c_{z}} \\
a_{5}=\frac{\varepsilon_{m f} u_{e}}{H\left[\left(1-\varepsilon_{m f}\right) a_{1}+\varepsilon_{m f}\right]} ; \quad B_{2}=\frac{\delta}{(1-\delta)\left(1-\varepsilon_{m f}+\varepsilon_{m f} / a_{1}\right)}\left(\frac{\alpha_{q}^{b e}}{\rho_{z} c_{z}}+\beta_{z}^{b e}\right) \\
B_{1 j}=\frac{\delta \beta_{g j}^{b e}}{(1-\delta) \varepsilon_{m f}} ; \quad B_{3 j}=\frac{H}{u_{b}} \beta_{g j}^{b e}, j=\mathrm{A}, \mathrm{P} \\
Q_{1}=\frac{a_{q} k_{q}}{\left(1-\varepsilon_{m f}+\varepsilon_{m f} / a_{1}\right) \rho_{z} c_{z}} ; \quad Q_{2}=\frac{H}{u_{b}} \frac{a_{q} k_{q}}{\rho_{z} c_{z}}
\end{gathered}
$$

Additional details concerning determination of the hydrodynamic parameters of the bed and mass and heat transfer coefficients between the emulsion phase and bubble phase can be found in (Bizon, 2016).

In case of the single chemical reaction it can be assumed that $k_{02}=0$, then the dynamics of the reactor is described by the system of two differential equations, i.e. Eqs. (2) and (4).

Table 1. Values of the model parameters used in numerical simulations

\begin{tabular}{|c|c|c|c|}
\hline No & Parameter & Value & Dimension \\
\hline 1 & $a_{q} k_{q}$ & $0.04-0.16$ & $\mathrm{~kW} / \mathrm{m}^{3} \cdot \mathrm{K}$ \\
\hline 2 & $c_{g}$ & 1.0 & $\mathrm{~kJ} / \mathrm{kg} \cdot \mathrm{K}$ \\
\hline 3 & $c_{z}$ & 0.8 & $\mathrm{~kJ} / \mathrm{kg} \cdot \mathrm{K}$ \\
\hline 4 & $d_{z}$ & $2.0 \times 10^{-4}$ & $\mathrm{~m}$ \\
\hline 5 & $E_{1}$ & $7.0 \times 10^{4}$ & $\mathrm{~kJ} / \mathrm{kmol}$ \\
\hline 6 & $\Delta_{1}$ & $-4.0 \times 10^{5}$ & $\mathrm{~m}$ \\
\hline 7 & $H_{m f}$ & $1.0-1.2$ & $1 / \mathrm{s}$ \\
\hline 8 & $k_{01}$ & $4.0 \times 10^{5}$ or $1.0 \times 10^{7}$ & $\mathrm{~K}$ \\
\hline 9 & $T_{f}$ & 450 & $x_{E}$ \\
\hline 10 & $T_{q}$ & 300 or 320 & $x_{k}$ \\
\hline 11 & $x_{E}, x_{\Delta h}$ & 1.5 & - \\
\hline 12 & $x_{k}$ & 1.0 & $\mathrm{~kg} / \mathrm{m}^{3}$ \\
\hline 13 & $y_{\mathrm{A} f}$ & 0.1 & $\mathrm{~kg} / \mathrm{m}^{3}$ \\
\hline 14 & $\rho_{g}$ & 0.7 & 2300 \\
\hline 15 & $\rho_{z}$ & & \\
\hline
\end{tabular}




\section{RESULTS AND DISCUSSION}

Values of the parameters used in numerical simulations both for the single chemical reaction and for the system of consecutive reactions are reported in Table 1 . The values were selected based on the analysis of typical catalytic processes (Balakotaiah and West, 2014; Westerink and Westertrep, 1992). Symbols $x_{E}, x_{\Delta h}$, and $x_{k}$ refer, respectively, to a ratio of activation energies, enthalpies and frequency coefficients in Arrhenius equation for the consecutive reaction, namely

$$
x_{E}=\frac{E_{2}}{E_{1}}, \quad x_{\Delta h}=\frac{\Delta h_{2}}{\Delta h_{1}}, \quad x_{k}=\frac{k_{02}}{k_{01}}
$$

A home-made code for continuation of the solution branches based on a local parameterisation (Seydel, 1994) was developed and used to determine the parametric dependency of the state variables and to characterize the structure of steady states of the fluidised-bed reactor. This is permitted to avoid application of complex continuation software, as recommended also in the reference (Berezowski, 2010).
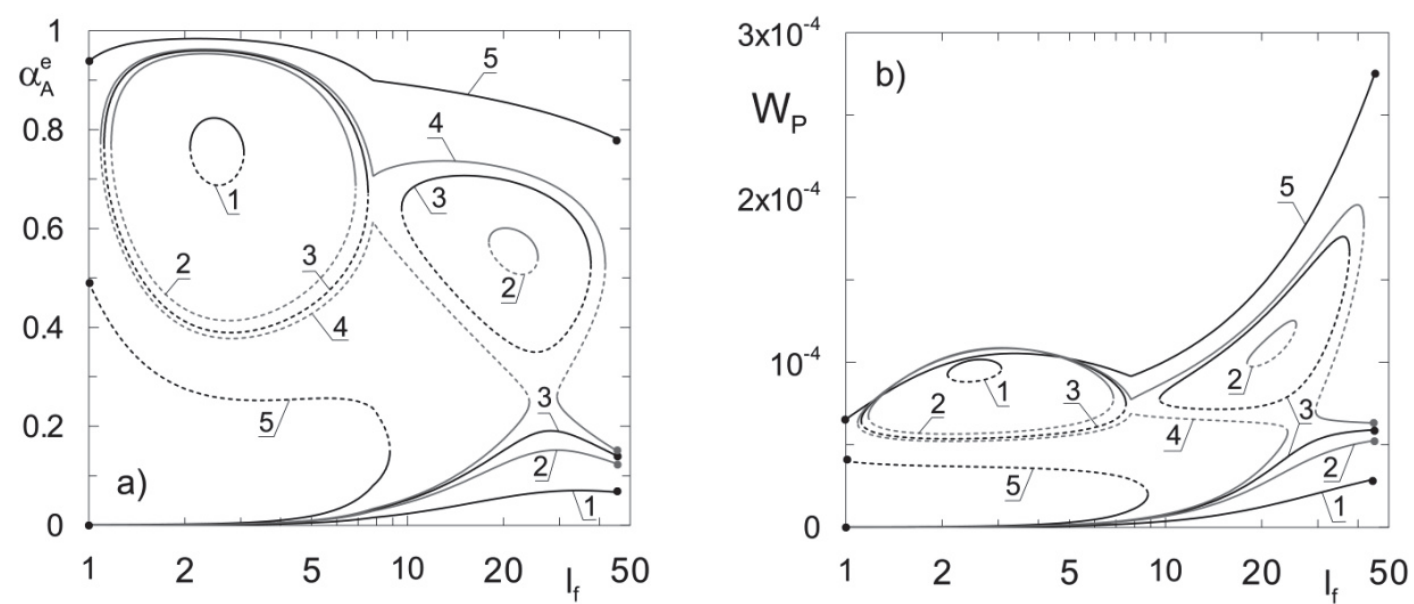

Fig. 1. Steady-state branches (a) and process yield $W_{\mathrm{P}}$ (b) for a single chemical reaction with respect to $l_{f}$ and $a_{q} k_{q} ; a_{q} k_{q}=\{1-0.16 ; 2-0.129 ; 3-0.125 ; 4-0.123 ; 5-0.1\} \mathrm{kW} / \mathrm{m}^{3} \cdot \mathrm{K} ; H_{m f}=1.2 \mathrm{~m}, T_{f}=450 \mathrm{~K}$; $T_{q}=300 \mathrm{~K}(\bullet-$ boundaries of the fluidised-bed existence)

Figure 1 shows steady-state characteristics of a catalytic fluidised-bed reactor for a single irreversible exothermic reaction with respect to a fluidisation ratio $l_{f}$ and for different values of a parameter $a_{q} k_{q}$. The parameter $a_{q} k_{q}$ is associated with the heat transfer between the fluidised-bed and a cooling medium. When $a_{q} k_{q}=0$, then the reactor is adiabatic. Solid lines indicate stable steady states, while dashed lines indicate unstable steady states. Values of a conversion degree of the reactant A, i.e. $\alpha_{\mathrm{A}}$, (Fig. 1a) are determined as follows

$$
\alpha_{\mathrm{A}}=\frac{C_{\mathrm{A} f}-C_{\mathrm{A}}}{C_{\mathrm{A} f}}=1-\eta_{\mathrm{A}} \frac{T^{e}}{T_{f}}
$$

whereas the process yield $W_{\mathrm{P}}$ (Fig. 1b) for the single chemical reaction is defined as

$$
W_{\mathrm{P}}=\frac{v_{\mathrm{P}}}{\left|v_{\mathrm{A}}\right|} u_{0} C_{\mathrm{A} f} \cdot \bar{\alpha}_{\mathrm{A}}(1)
$$

where $\bar{\alpha}_{\mathrm{A}}(1)$ is an average conversion degree of A leaving the fluidised-bed.

Two first type isolas arising above the lower stable steady state were detected at relatively high values of the parameter $a_{q} k_{q}$. The complexity of the steady-state structure of the reactor is a result of a 
coupling of chemical reaction with mass and heat transfer processes between the bubbles and the emulsion phase. It can be observed that stable fragments (branches 2 and 3 in Fig. 1b) of the right isola located in the region of high values of the fluidisation $l_{f}$ are characterized by higher yield of the process $W_{\mathrm{P}}$.

The location of the isolas in the bifurcation diagram depends on the type of a chemical process carried out in the fluidised-bed. The steady-state structure of the fluidised-bed corresponding to the process described by two consecutive chemical reactions is shown in Fig. 2. In such a case the process yield with respect to the product $\mathrm{P}, W_{\mathrm{P}}$ is calculated as

$$
W_{\mathrm{P}}=\frac{v_{\mathrm{P}}}{\left|v_{\mathrm{A}}\right|} u_{0} C_{\mathrm{A} f} \cdot \bar{\alpha}_{\mathrm{P}}(1)
$$

where a dimensionless concentration of the product $\mathrm{P}$ defined with respect to the feed temperature is as follows

$$
\alpha_{\mathrm{P}}=\frac{C_{\mathrm{P}}-C_{\mathrm{P} f}}{C_{\mathrm{A} f}}=1-\eta_{\mathrm{P}} \frac{T^{e}}{T_{f}}
$$

In case of the system of two reactions in series, fivefold steady states can occur. For the catalytic process analysed in this study for a certain set of the model parameters the branches of steady states may take a form of multiple isolas. This is confirmed by the results presented in Fig. 2. Unlike the single chemical reaction, the isolas are located in the same range of the parameter, i.e. one isola is located above the other. Therefore, for such isolas the concept of co-existence was introduced.
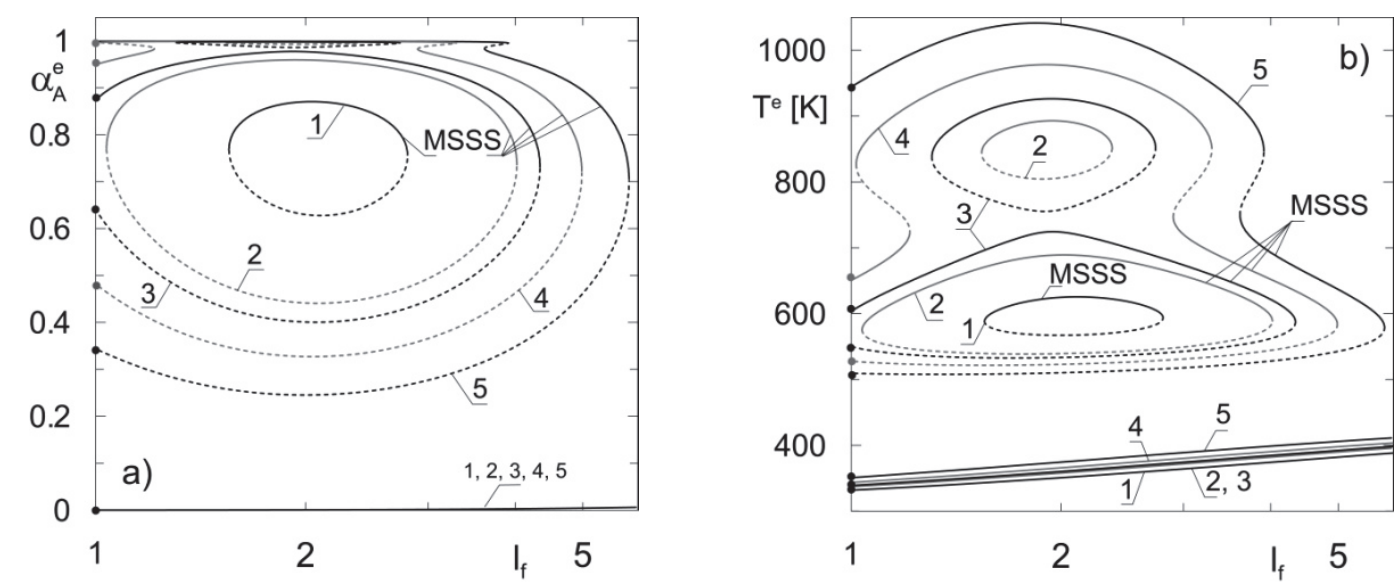

Fig. 2. Steady-state branches for a system of consecutive reactions with respect to $l_{f}$ and $a_{q} k_{q}$; $a_{q} k_{q}=\{1-0.076 ; 2-0.062 ; 3-0.058 ; 4-0.05 ; 5-0.04\} \mathrm{kW} / \mathrm{m}^{3} \cdot \mathrm{K} ; H_{m f}=1 \mathrm{~m}, T_{f}=450 \mathrm{~K} ;$

$T_{q}=300 \mathrm{~K}(\bullet-$ boundaries of the fluidised-bed existence $)$

To evaluate the influence of the bed hydrodynamics and the intensity of heat exchange on the steadystate structure globally, catastrophic sets in the plane $\left(l_{f}, a_{q} k_{q}\right)$ were then determined. The diagrams presented in Fig. 3 are the result of a two-parameter continuation (Seydel, 1994) of the turning points from Fig. 1 and Fig. 2. For a better understanding of the phenomenon of generation and disappearance of multiple isolas, the ranges of the parameter $a_{q} k_{q}$ in which they emerge are delimited with dashed lines.

In case of the single chemical reaction (Fig. 3a) and relatively high values of $a_{q} k_{q}$ the system has a unique stable solution branch. It is characterized by a low conversion degree and thus by a low yield of the reactor $W_{\mathrm{P}}$. Maxima of the upper curve marked with letters A and B (Fig. 3a) are referred to as the isola centres. They correspond to the values of the parameters $a_{q} k_{q}$ and $l_{f}$ at which the isolas are 
generated being an origin of a region of the triple steady states. Diminution of the heat exchange intensity represented by the product $a_{q} k_{q}$ leads to enlargement of the isolas and thus extension of the region of the triple steady states. At the value of $a_{q} k_{q}$ corresponding to the point $\mathrm{C}$ (Fig. $3 \mathrm{a}$ ) the two isolas merge, then further diminution of $a_{q} k_{q}$ leads to their coalescence with the lower stable branch of steady states.
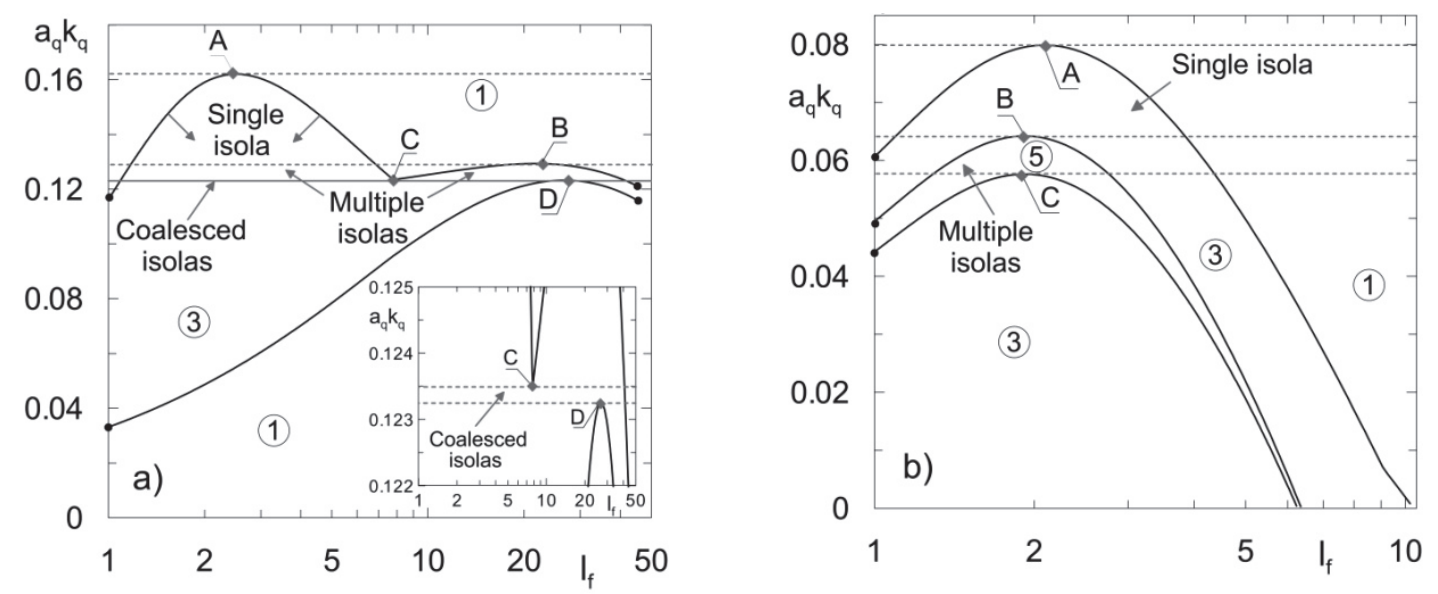

Fig. 3. Catastrophic sets in the plane $\left(l_{f}, a_{q} k_{q}\right)$ for a single chemical reaction (a) and for a system of consecutive reactions (b); $T_{f}=450 \mathrm{~K}, T_{q}=300 \mathrm{~K}$ (numbers indicate the number of steady states)

Figure $3 \mathrm{~b}$ shows a steady-state structure in the plane $\left(l_{f}, a_{q} k_{q}\right)$ determined for the reactor in which a system of two consecutive chemical reactions takes place. Extrema of the curves marked with A and B (Fig. 3b) correspond, respectively, to the parameters for which isolas occur in the system. The point $\mathrm{C}$ (Fig. 3b) corresponds to the process conditions at which the isolas coalesce. The region of the triple steady states resulting from the coalescence of the isolas, i.e. the area located under the lower curve, corresponds to solutions disadvantageous from the technical point of view. In this region the system only has the lower steady state that does not guarantee the autothermicity of the process and the upper steady state corresponding to a very high conversion but also to an extremely high temperature, thus it characterised by a very low yield of the process $W_{\mathrm{P}}$.

Taking into account that the model of the reactor depends on some parameters whose values can change dynamically during the process, both intentionally and due to the external disturbances, the appearance both of a single and multiple isolas may strongly influence the process dynamics. Due to the observed strong impact of the fluidisation ratio $l_{f}$ onto the steady-state structure, the influence of its variation onto the reactor dynamics was evaluated. The variation of $l_{f}$ may be intentional, i.e. it can take place during the reactor start-up, when the fluidisation ratio is dynamically increased to reach a given target value. It can also be induced by the process kinetics, which is the case for non-equimolar processes (Abba et al., 2002) characterized by a variable volumetric flow rate of a fluidising medium.

Figure 4 shows various scenarios of the variation of the fluidization ratio $l_{f}$ (Fig. $\left.4 \mathrm{a}\right)$ with corresponding dynamical responses of the reactor (Fig. $4 \mathrm{~b}$ and Fig. $4 \mathrm{c}$ ) in which a single irreversible reaction takes place. Two starting values of the fluidization ratio corresponding to the time instant $t=0$ were used in the simulations, i.e. $l_{f 0}=2$ and $l_{f 0}=5.5$. For all the evaluated cases the initial conditions of the dynamic model of the reactor were set to $\alpha_{\mathrm{A} 0}^{e}=0$ that corresponds to $\eta_{\mathrm{A} 0}^{e}=1$ and $T_{0}^{e}=450 \mathrm{~K}$. A target value of the fluidization ratio was set to $l_{f}=25$. Trajectories $l_{f}(t), T^{e}(t)$ and $W_{\mathrm{P}}(t)$ corresponding to $l_{f 0}=2$ labelled with numbers 1 and 2 in Fig. 4 correspond, respectively, to fast and slow variations of the fluidisation ratio (Fig. 4a). In both cases the reactor eventually reaches the upper steady state. Yet, in case of a low rate of increase (curves marked with 2 in Fig. 4) the trajectory $T^{e}(t)$ exhibits a substantial maximum. This phenomenon can be dangerous from the technological point of view as it can lead, for 
instance, to the thermal deactivation of the catalyst. Moreover, it also causes a significant decrease in the instantaneous yield of the process (Fig. 4c).
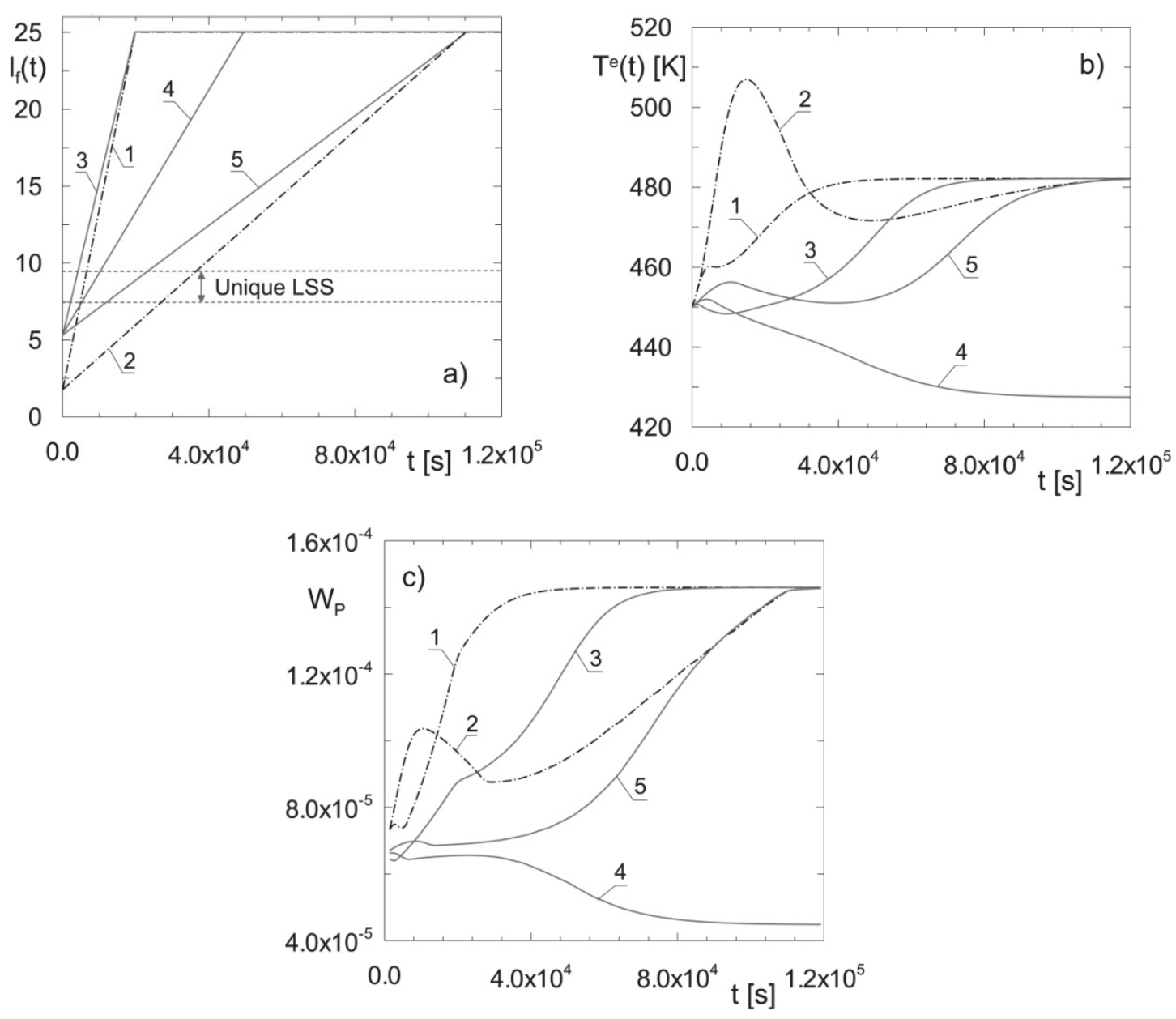

Fig. 4. Dynamic variation of $l_{f}$ (a) and corresponding responses of the reactor (b-c) for a single chemical reaction for $a_{q} k_{q}=0.125 \mathrm{~kW} / \mathrm{m}^{3} \cdot \mathrm{K} ; H_{m f}=1.2 \mathrm{~m} ; T_{f}=450 \mathrm{~K} ; T_{q}=300 \mathrm{~K}$

The dynamical response of the reactor to the variation of fluidization ratio changes when $l_{f 0}=5.5$. In such a case the fast increase of the fluidization ratio value leads to the convergence of the trajectory at the upper steady state (curves labelled with 3 in Fig. 4). For a lower rate of the variation of $l_{(}(t)$ the trajectory converges at the lower steady state as shown by curves marked with 4 in Fig. 4. However, further prolongation of the time interval in which the process conditions change (curves labelled with 5 in Fig. 4) again results in the convergence at the upper steady state. Such a phenomenon is associated with the fact that if the rate of increase of the fluidization ratio is low then in the initial stage of the process the bed heats up enough to maintain a sufficient level of the temperature, even when operating transitorily in the interval of $l_{f}$ for which the reactor has only the lower steady state.

Three scenarios of the increase of the fluidization ratio were considered for a system of reactions in series, starting at the minimal value $l_{f}=1.01$, and ending at the target value coinciding with a high yield of the reactor, that is at $l_{f 0}=3.5$ (Fig. 5a). A dangerous rise of the bed temperature is observed at a low rate of the variation of $l_{f}$ (curve labelled with 3 in Fig $5 \mathrm{~b}$ ). This is due to the fact that for the assumed value of the parameter $a_{q} k_{q}=0.05$ two merged isolas of the second type were detected in the reactor and only two stable steady states exist in the interval $l_{f}=[1.214,2.899]$ (branches marked with 4 in Fig. 2), with the upper one being disadvantageous from the process point of view. Thus, exceeding the value $l_{f}=1.214$ corresponding to the first turning point, results in the temporary attraction of the trajectories by the upper steady state. At the higher cooling intensity of the reactor, i.e. for $a_{q} k_{q}=0.058$ at which the 
multiple isolas were detected (branches denoted with number 3 in Fig. 2) this risk does not exist. In such a case the trajectories converge directly at the middle steady state (Fig. 5c). If the initial conditions belong to the basins of attraction of the middle steady state then in the region of the occurrence of multiple isolas the dynamical variation of $l_{f}$ always leads to the convergence at this steady state.
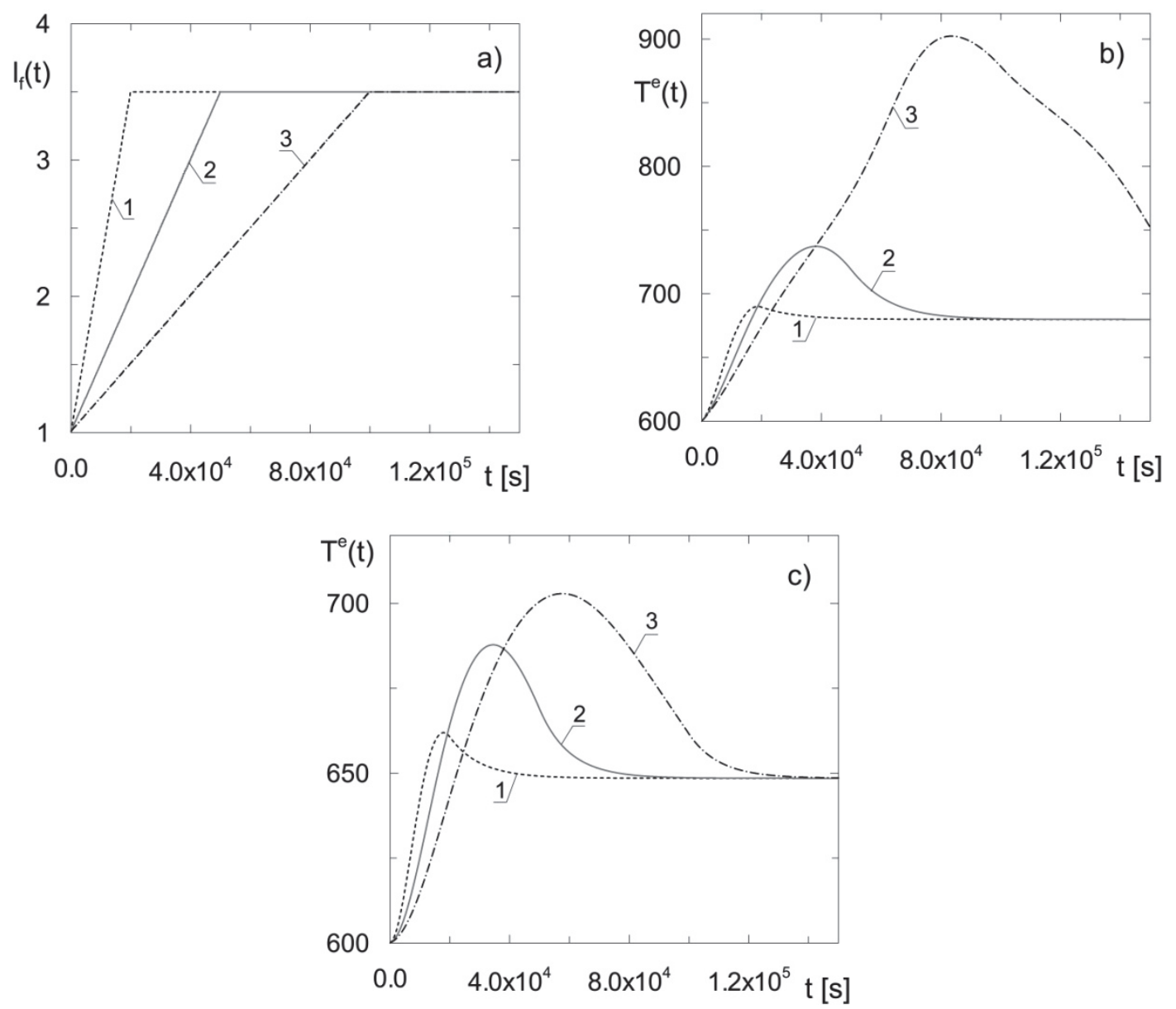

Fig. 5. Dynamic variation of $l_{f}$ (a) and corresponding responses of the reactor of a system of consecutive chemical reactions for $a_{q} k_{q}=0.05 \mathrm{~kW} / \mathrm{m}^{3} \cdot \mathrm{K}$ (b) and for $a_{q} k_{q}=0.058 \mathrm{~kW} / \mathrm{m}^{3} \cdot \mathrm{K}(\mathrm{c}) ; H_{m f}=1.2 \mathrm{~m} ; T_{f}=450 \mathrm{~K} ; T_{q}=300 \mathrm{~K}$
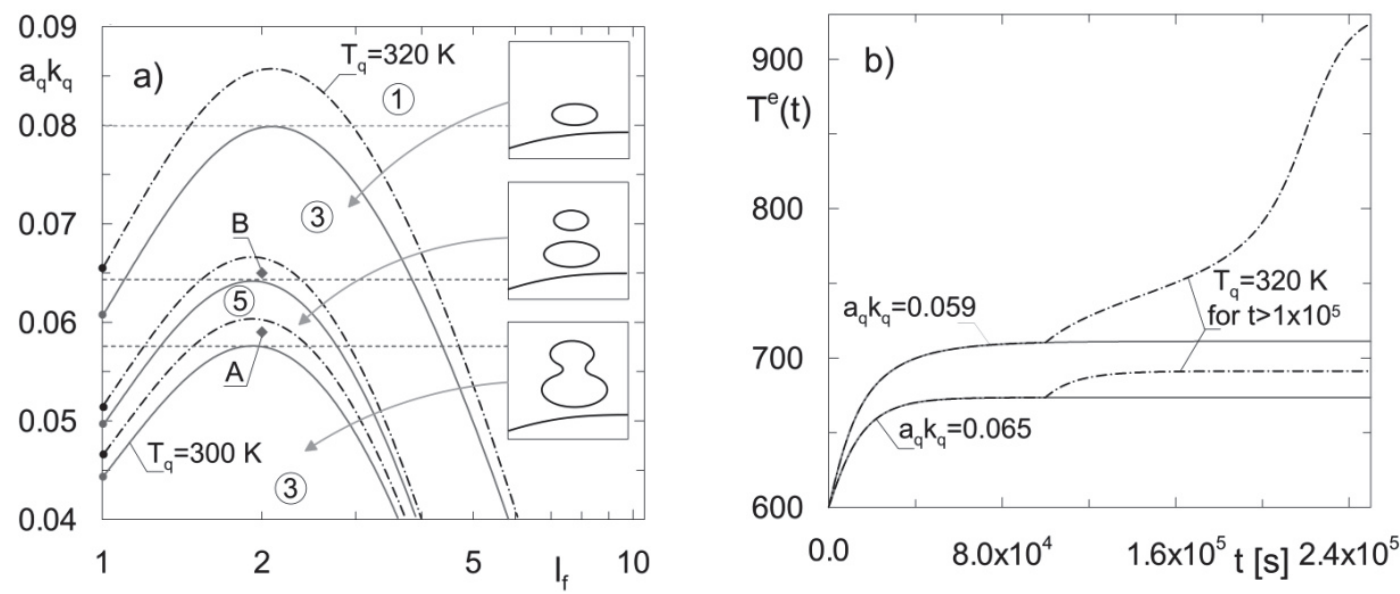

Fig. 6. Catastrophic sets in the plane $\left(l_{f}, a_{q} k_{q}\right)$ for a system of consecutive reactions for $T_{q}=300 \mathrm{~K}$ (solid line) and for $T_{q}=320 \mathrm{~K}$ (dashed line) (a) and the reactor response to a step disturbance of a cooling medium temperature $T_{q}(\mathrm{~b}) ;\left(T_{f}=450 \mathrm{~K}, l_{f}=2\right.$; numbers indicate the number of steady states) 
All the above analysis is focused on the influence of variation of the fluidisation ratio onto the reactor dynamics. However, other parameters of the model may also vary during the process, both intentionally and uncontrollably. Figure 6 shows the impact of the cooling medium temperature $T_{q}$ onto the stationary and dynamical properties of the reactor for a system of reactions in series. It can be observed (Fig. 6a) that the increase of the temperature $T_{q}$ from 300 to $320 \mathrm{~K}$ does not change the steady-state structure fundamentally. There is only a quantitative shift of the regions of a given multiplicity in the plane $\left(l_{f}, a_{q} k_{q}\right)$. However, it appears that the shift of the regions may have a strong impact on the reactor dynamics in case of step disturbances of the temperature $T_{q}$.

Let us assume that the process is carried out at a fixed intensity of a heat exchange and at a constant value of the fluidization ratio. In particular, a pair of parameter values $a_{q} k_{q}=0.059$ and $l_{f}=2$ corresponding to point A in Fig. 6a is considered. In such a case, at the temperature $T_{q}=300 \mathrm{~K}$ fivefold steady states occur in the reactor, with the middle and upper steady states located on the second type isolas. The dynamic properties of the reactor in the neighbourhood of point A can be learnt by the analysis of the trajectories of the reactor subject at some time instant $t_{0}$ to a step disturbance of the cooling medium temperature $T_{q}$. The trajectories of the temperature of emulsion $T^{e}(t)$ are shown in Fig. $6 \mathrm{~b}$. It can be observed that the dynamic response of the reactor and its performance at a new steady state depends on the choice of the parameter characterizing the intensity of the heat exchange, i.e. $a_{q} k_{q}$. Let us consider the first case in which $a_{q} k_{q}=0.059$ corresponds to the point A in Fig. 6a. If a step disturbance of the cooling medium temperature up to $T_{q}=320 \mathrm{~K}$ takes place at a certain time instant $t_{0}$ then a substantial rise of the temperature in the reactor is detected (dashed line in Fig. 6b). This is due to the fact that for this value of $T_{q}$ the point A belongs to the triple steady-state region arisen from the coalescence of two isolas (Fig. 6a), therefore the trajectories are attracted by the upper steady state that is disadvantageous from the technological point of view.

The situation changes at $a_{q} k_{q}=0.065$ and $l_{f}=2$ corresponding to the point $\mathrm{B}$ in Fig. 6a. Then, at $T_{q}=300 \mathrm{~K}$ the reactor has three steady states resulting from the first isola above the lower steady state. Stable fragments of the isola correspond to moderate temperatures and thus satisfactory values of the process yield $W_{\mathrm{P}}$. The trajectories shown in Fig. $6 \mathrm{~b}$ confirm the convergence at the technologically advantageous stable steady state. There is no qualitative change of the solution in the event of step increase of the cooling medium temperature at a certain $t_{0}$, that is after stabilization of the process in the apparatus, to the value $T_{q}=320 \mathrm{~K}$. As a result of the shift of the boundaries of the multiplicity regions mentioned before, the point B belongs then to the fivefold steady-state area. Despite a relatively large magnitude of the disturbance the trajectories are not attracted by the other steady state, i.e. the lower or upper one.

\section{CONCLUSIONS}

An influence of the selected operating parameters onto steady-state characteristics and dynamics of a catalytic fluidized bed reactor was presented. The relationship between the steady-state characteristics of the reactor, in particular of the region of multiple isolas, and its dynamics was evaluated.

For a single chemical reaction and for a system of two consecutive reactions the model was shown to exhibit an untypical steady-structure having a form of multiple isolas. Depending on the process kinetics, a relative location of the isolas in the bifurcation diagram can be different, both from a mathematical and technological point of view.

It was demonstrated that for the single exothermic reaction two isolas of steady states can coexists in different intervals of the bifurcation parameter. They can be classified as the first type isolas. Quite distinct steady-state characteristics was observed for the system of two consecutive reactions. Due to the higher complexity of the process a region of fivefold steady states may occur. Hence, there is a 
possibility of the generation of two isolas located one above the other. They were called the second type isolas.

The analysis performed in the paper shows that the occurrence of multiple isolas of steady states can have serious dynamical and technological consequences. It is particularly important in the case of variation of a process parameter that influences the bed hydrodynamics, in this case the fluidization ratio $l_{f}$. For the single chemical reaction, in order to maintain the performance of the reactor at a high level, a dynamical change of $l_{f}$ has to be done fast enough or slow enough, depending on the initial conditions in the apparatus. Therefore the choice of the strategy of variation of this parameter requires prior knowledge of the reactor dynamics. As demonstrated in this work, running the process in the region of the isolas detected for the system of consecutive reactions can also have serious technological consequences.

It was demonstrated that in the region of the second type multiple isolas the reactor's response to a step disturbance of the cooling medium temperature depends on the heat exchanger size. For the mismatched size of the heat exchanger the disturbance of the cooling medium temperature may lead the reactor to the disadvantageous steady state characterized by high temperature and low performance.

The results presented in this work confirm that the knowledge of steady-state and dynamic properties of the reactor is indispensable to design and to run exothermal processes in systems characterized by such a complex steady-state structure.

\section{SYMBOLS}

$\begin{array}{ll}a_{i} & \text { model parameter } \\ a_{q} & \text { heat transfer area per unit volume of a bed, } \mathrm{m}^{-1} \\ B_{i}, B_{i j} & \text { model parameter determining the interphase mass and heat transfer } \\ c_{g}, c_{z} & \text { specific heat of gas and solid particles, } \mathrm{kJ} \cdot \mathrm{kg}^{-1} \cdot \mathrm{K}^{-1} \\ C_{j} & \text { concentration of } j^{\text {th }} \text { reactant, } \mathrm{kmol} \cdot \mathrm{m}^{-3} \\ d_{z} & \text { catalyst pellet diameter, } \mathrm{m} \\ E_{i} & \text { activation energy of } i^{\text {th }} \text { chemical reaction, } \mathrm{kJ} \cdot \mathrm{kmol}^{-1} \\ h & \text { height above the distributor, } \mathrm{m} \\ \Delta h_{i} & \text { enthalpy of } i^{\text {th }} \text { chemical reaction, } \mathrm{kJ} \cdot \mathrm{kmol}^{-1} \\ H & \text { total height of a fluidized bed, } \mathrm{m} \\ k_{q} & \text { overall heat transfer coefficient, } \mathrm{kJ} \cdot \mathrm{m}^{-2} \cdot \mathrm{s}^{-1} \cdot \mathrm{K}^{-1} \\ k_{i} & \text { rate constant of the } i^{\text {th }} \text { chemical reaction } \\ k_{0 i} & \text { frequency coefficient in Arrheniusa eqaution of } i^{\text {th }} \text { chemical reaction, } \mathrm{s}^{-1} \\ l_{f} & \text { fluidization ratio } \\ Q_{i} & \text { model parameters determining intensity of heat transfer } \\ r_{i} & \text { rate of } i^{\text {th }} \text { chemical reaction, } \mathrm{kmol} \cdot \mathrm{m}^{-3} \cdot \mathrm{s}^{-1} \\ \widetilde{r}_{i} & \text { rate of } i^{\text {th }} \text { chemical reaction in a function of dimensionless concentration } \eta_{j}, \mathrm{~s}^{-1} \\ R & \text { gas constant, } \mathrm{kJ} \cdot \mathrm{kmol}^{-1} \cdot \mathrm{K}^{-1} \\ t & \text { time, } \mathrm{t} \\ T & \text { temperature, } \mathrm{K} \\ u & \text { velocity, } \mathrm{m} \cdot \mathrm{s}^{-1} \\ W_{P} & \text { yield of a process with respect to product } \mathrm{P}, \mathrm{kmol} \cdot \mathrm{m}^{-2} \cdot \mathrm{s}^{-1} \\ y_{j} & \text { molar fraction of } j^{\text {th }} \mathrm{reactant} \\ z & \text { dimensionless height above distributor }\end{array}$

Greek symbols

$\alpha_{A} \quad$ conversion degree of reactant A 
$\alpha_{P} \quad$ dimensionless concentration of product $\mathrm{P}$ defined with respect to the feed temperature

$\alpha_{q}^{i j}$ heat exchange coefficient between phase $i$ and $j, \mathrm{~kJ} \cdot \mathrm{m}^{-3} \cdot \mathrm{s}^{-1} \cdot \mathrm{K}^{-1}$

$\beta_{g k}^{i j}$ gas exchange coefficient of $k^{\text {th }}$ reactant between phases $i$ and $j, \mathrm{~s}^{-1}$

$\beta_{z}^{i j}$ solid exchange coefficient between phases $i$ and $j, \mathrm{~s}^{-1}$

$\delta \quad$ volumetric fraction of bubbles in a bed

$\varepsilon_{m f} \quad$ void fraction at minimum fluidization

$v_{j} \quad$ stoichiometric coefficient of the $j^{\text {th }}$ reactant

$\eta_{j} \quad$ dimensionless concentration of $j^{\text {th }}$ reactant

$\rho_{g}, \rho_{z} \quad$ gas and sold particles density, $\mathrm{kg} \cdot \mathrm{m}^{-3}$

\section{Subscripts}

$A, P \quad$ refers to reactant $\mathrm{A}$ and product $\mathrm{P}$

$b \quad$ refers to bubbles

e refers to emulsion

$f \quad$ refers to feed stream

mf refers to minimum fluidisation condition

$q \quad$ refers to cooling medium

\section{Superscript}

$b, c, e \quad$ refers to bubble, clouds and emulsion phase, respectively

\section{REFERENCES}

Abba I.A., Grace J.R., Bi H.T., 2002. Variable-gas-density fluidized bed reactor model for catalytic processes. Chem. Eng. Sci., 57, 4797-4807. DOI: 10.1016/S0009-2509(02)00289-0.

Ajbar A., Alhumazi K., Elnashaie, S.S.E.H., 2001. Classification of static and dynamic behaviour in a fluidizedbed catalytic reactor. Chem. Eng. J., 84, 503-516. DOI: 10.1016/S1385-8947(00)00373-9.

Balakotaiah V., West D.H., 2014. Thermal effects and bifurcations in catalytic partial oxidations. Curr. Opin, Chem, Eng., 5, 68-77. DOI: 10.1016/j.coche.2014.05.002.

Berezowski M., 2000. Method of determination of steady-state diagrams of chemical reactors. Chem. Eng. Sci., 55, 4291-4295. DOI: 10.1016/S0009-2509(00)00058-0.

Berezowski M., 2002. Złożona struktura stanów stacjonarnych układu równolegle połączonych reaktorów zbiornikowych. Inż. Chem. Proc., 23, 415-423.

Berezowski M., 2010. The application of the parametric continuation method for determining steady state diagrams in chemical engineering. Chem. Eng. Sci., 65, 5411-5414. DOI: 10.1016/j.ces.2010.07.003.

Bizon K., 2016. Autothermicity, multiplicity, yield and selectivity of catalytic processes in a polytropic fluidized bed reactor. Chem. Eng. J., 288, 834-844. DOI: 10.1016/j.cej.2015.12.061.

Pinto J.C., Ray W.H., 1995. The dynamic behavior of continuous solution polymerization reactors - VIII. A full bifurcation analysis of a lab-scale copolymerization reactor. Chem. Eng. Sci., 50, 1041-1056. DOI: 10.1016/j.cej.2015.12.061.

Seydel R., 1994. Practical bifurcation and stability analysis. From equilibrium to chaos. Springer-Verlag, New York.

Tabiś B., 2001. Methanol synthesis in a fluidized-bed reactor coupled with an external heat exchanger. The effect of feedback deformation. Chem. Eng. J., 83, 191-200. DOI: 10.1016/S1385-8947(00)00254-0.

Uppal A., Ray W.H., Poore A.B., 1976. The classification of the dynamic behavior of continuous stirred tank reactors - Influence of reactor residence time. Chem. Eng. Sci., 31, 205-214. DOI: 10.1016/00092509(76)85058-0.

Westerink E.J., Westerterp K.R., 1990. Stable design and operation of catalytic fluidized-bed reactors for multiple reactions: Uniqueness and multiplicity. Chem. Eng. J., 45, 317-332. DOI: 10.1016/0009-2509(90)87103-Y.

Zahn V.M, 2012. Adiabatic simulated moving bed reactor - principle, nonlinear analysis and experimental demonstration. Shaker Verlag GmbH, Herzogenrath. 


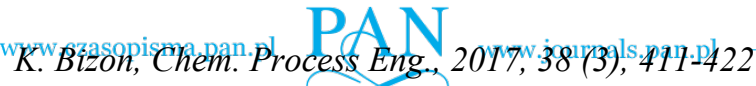

Received 3 October 2016

Received in revised form 10 July 2017

Accepted 13 July 2017 